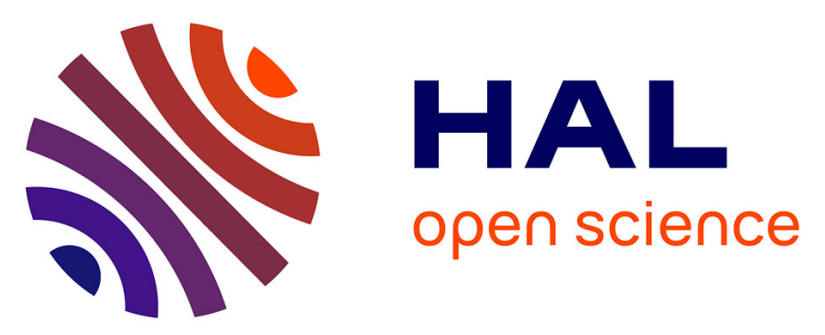

\title{
On the multi-scale description of micro-structured fluids composed of aggregating rods
}

Marta Perez, Adrien Scheuer, Emmanuelle Abisset-Chavanne, Amine Ammar, Francisco Chinesta, Roland Keunings

\section{- To cite this version:}

Marta Perez, Adrien Scheuer, Emmanuelle Abisset-Chavanne, Amine Ammar, Francisco Chinesta, et al.. On the multi-scale description of micro-structured fluids composed of aggregating rods. Continuum Mechanics and Thermodynamics, 2019, 31 (4), pp.955-967. 10.1007/s00161-018-0659-1 . hal$02163613 \mathrm{v} 2$

\section{HAL Id: hal-02163613 \\ https://hal.science/hal-02163613v2}

Submitted on 28 Jan 2020

HAL is a multi-disciplinary open access archive for the deposit and dissemination of scientific research documents, whether they are published or not. The documents may come from teaching and research institutions in France or abroad, or from public or private research centers.
L'archive ouverte pluridisciplinaire HAL, est destinée au dépôt et à la diffusion de documents scientifiques de niveau recherche, publiés ou non, émanant des établissements d'enseignement et de recherche français ou étrangers, des laboratoires publics ou privés. 


\title{
Marta Perez • Adrien Scheuer • Emmanuelle Abisset-Chavanne • Amine Ammar • Francisco Chinesta • Roland Keunings \\ On the multi-scale description of micro-structured fluids composed of aggregating rods
}

\begin{abstract}
When addressing the flow of concentrated suspensions composed of rods, dense clusters are observed. Thus, the adequate modelling and simulation of such a flow requires addressing the kinematics of these dense clusters and their impact on the flow in which they are immersed. In a former work, we addressed a first modelling framework of these clusters, assumed so dense that they were considered rigid and their kinematics (flow-induced rotation) were totally defined by a symmetric tensor $\mathbf{c}$ with unit trace representing the cluster conformation. Then, the rigid nature of the clusters was relaxed, assuming them deformable, and a model giving the evolution of both the cluster shape and its microstructural orientation descriptor (the so-called shape and orientation tensors) was proposed. This paper compares the predictions coming from those models with finer-scale discrete simulations inspired from molecular dynamics modelling.
\end{abstract}

Keywords Aggregate - Cluster - Jeffery's equation - Non-ellipsoidal particles · Concentrated suspensions · Multi-scale modelling

Communicated by Francesco dell'Isola.

M. Perez

ESI Group Centre Composites R\&D, Aero Business Center Aeroparc, 25 Avenue Marcel ISSARTIER Boite 20005,

33702 Mrignac Cedex, France

E-mail: marta.perezmiguel@esi-group.com

E. Abisset-Chavanne - A. Scheuer

ESI Group Chair, ICI, Centrale Nantes, 1 Rue de la Noe, 44300 Nantes, France

E-mail: Emmanuelle.Abisset-chavanne@ec-nantes.fr

A. Scheuer

E-mail: adrien.scheuer@ec-nantes.fr; adrien.scheuer@uclouvain.be

A. Ammar

LAMPA, ENSAM Angers, 2 Boulevard du Ronceray, BP 93525, 49035 Angers Cedex 01, France

E-mail: Amine.AMMAR@ensam.eu

F. Chinesta $(\varangle)$

ESI Group Chair, PIMM, ENSAM ParisTech, 151 Boulevard de l'Hôpital, 75013 Paris, France

E-mail: Francisco.Chinesta@ensam.eu

R. Keunings · A. Scheuer

ICTEAM, Université catholique de Louvain, Bat. Euler, Av. Georges Lemaitre 4, 1348 Louvain-la-Neuve, Belgium

E-mail: Roland.Keunings@uclouvain.be 


\section{Introduction}

Suspensions involving rods are described at three different scales. At the microscopic scale, the motion of each rod is tracked using Jeffery's equation [28] and their impact on the fluid rheology evaluated from one of the available models [7,23-27,38]. At the mesoscopic scale, the individuality of particles is replaced by a distribution function giving the probability of finding a particle at a given position and time and having a given orientation. At that scale, usual physical coordinates (space and time) are complemented with some conformational coordinates $[9,20]$ (in the present case, the orientation described by the unit vector $\mathbf{p}$ ) in order to retain the main fluid features. Finally, at the macroscopic scale, only the moments of the distribution function are retained - the so-called orientation tensors - whose time evolution only involves the physical coordinates.

Moving from the microscopic to the mesoscopic scale is motivated by the computational complexity that the consideration of large population of interacting particles entails (as well as the particles-fluid interactions). In turn, mesoscopic descriptions are confronted to issues related to their multidimensional character and the associated inability of most experienced discretization techniques to address their simulation. For circumventing the curse of dimensionality, several techniques exist: (1) most of them are based on the used of computational particles $[10,13,17,18,37,41]$; (2) other procedures consider standard discretization techniques with adapted approximations [11,32]; (3) finally a third group consists of the use of separated representations proposed some years ago by the authors, with as main ingredient the so-called Proper Generalized Decomposition $[5,6,14,16,36]$. At the macroscopic scale, the equations governing the time evolution of the distribution moments usually involve closure approximations whose impact on the results is unpredictable $[12,29]$.

In [3], the flow model of a suspension composed of rods was established, in the dilute and semi-dilute regimes by considering nine structural bricks, three at each scale (microscopic, mesoscopic, and macroscopic). These three key bricks are: (1) the considered conformational variable, (2) the equation governing its time evolution, and (3) its contribution to the suspension stress.

When the concentration increases, particles tend to aggregate [33-35] creating a new microstructure with an intermediate scale. Indeed, instead of having rods dispersed into a fluid matrix, the flowing system is composed of dense rod aggregates immersed into the fluid matrix.

A first attempt to describe such flowing systems has been proposed in [3]. There, it was assumed that these dense clusters of rods are and remain rigid enough to evolve as a rigid solid leading to a cluster flow-induced kinematics depending on a single conformation tensor [3]. The main results will be summarized in Sect. 2. This framework was extended [8] by considering the flow-induced motion and deformation of deformable aggregates, in which orientation and shape time evolutions are described by using two conformation tensors. The corresponding model is also summarized in Sect. 2.

The present work only addresses the cluster kinematics, rheology will be addressed in a future work, because it requires formulating the behaviour within a generalized continuum framework, as considered when addressing other media with internal structure $[2,19,22,39]$.

While in the case of rigid cluster, we could validate the model using the existing analogy between tridumbbells and ellipsoids, in the case of deformable clusters, the flow-induced deformation will be validated by comparing the model predictions with direct numerical simulations inspired by molecular dynamicsMD—descriptions, including rods and their interactions.

After this introduction, Sect. 2 revisits the description of rigid and deformable clusters composed of rods. Then, in order to validate the predictions, Sect. 3 proposes a finer-scale discrete description inspired from molecular dynamics simulations. Finally, Sect. 4 presents and discusses some numerical results.

\section{Revisiting description of rigid and deformable clusters composed of rods}

This section summarizes the main elements involved in the modelling of rigid and deformable clusters, deeply described in $[3,8]$, respectively.

\subsection{Rigid clusters}

Let consider a 3D rigid cluster, sketched in Fig. 1, consisting of $N / 2$ rods $\mathcal{R}_{j}$ of length $2 L_{j}$. Each rod $\mathcal{R}_{j}$ is assumed containing two beads at its ends on which hydrodynamic forces apply. Thus, the cluster contains $N$ beads $\mathcal{B}_{i}, i=1, \ldots, N$. The location of each bead $\mathcal{B}_{i}$ with respect to the cluster centre of gravity $\mathbf{G}$ is represented by $S_{i} \mathbf{q}_{i}$, where $\mathbf{q}_{i}$ is the unit vector pointing from $\mathbf{G}$ to $\mathcal{B}_{i}$. 


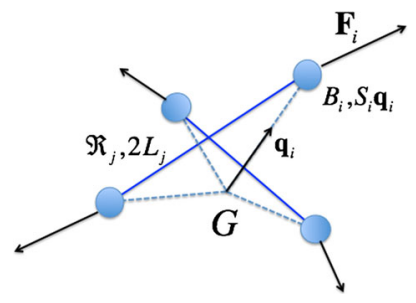

Fig. 1 Rigid cluster composed of rods

Brownian effects are neglected, and only hydrodynamic forces, assumed acting at each bead, are retained. Thus, on each bead located at position $S_{i} \mathbf{q}_{i}$ (Fig. 1) acts a force $\mathbf{F}_{i}$ proportional to the difference between the velocity of the unperturbed flow at the bead location, and the velocity of the bead itself:

$$
\mathbf{F}_{i}=\xi\left(\mathbf{V}_{0}+\nabla \mathbf{v} \cdot \mathbf{q}_{i} S_{i}-\mathbf{V}_{G}-\dot{\mathbf{q}}_{i} S_{i}\right),
$$

where $\xi$ is the friction coefficient, $\mathbf{v}$ the flow velocity field, $\mathbf{V}_{0}$ the fluid velocity at the cluster centre of gravity $\mathbf{G}$ and $\mathbf{V}_{G}$ the velocity of the cluster centre of gravity itself.

The equilibrium neglecting inertia terms implies

$$
\mathbf{0}=\sum_{i=1}^{i=N} \mathbf{F}_{i}=N\left(\mathbf{V}_{0}-\mathbf{V}_{G}\right)+\nabla \mathbf{v} \cdot\left(\sum_{i=1}^{i=N} S_{i} \mathbf{q}_{i}\right)-\left(\sum_{i=1}^{i=N} S_{i} \dot{\mathbf{q}}_{i}\right),
$$

where both sums in Eq. (2) vanish, the first one as a direct consequence of the definition of the centre of gravity, and the second because the cluster is assumed rigid. Thus, Eq. (2) becomes

$$
\mathbf{V}_{0}=\mathbf{V}_{G},
$$

implying that the cluster centre of gravity is moving with the fluid velocity at that position.

The torque created by the forces applied on bead $\mathcal{B}_{i}$ is given by

$$
\mathbf{M}_{i}=S_{i} \mathbf{q}_{i} \times \mathbf{F}_{i},
$$

whose resultant must also vanish:

$$
\sum_{i=1}^{i=N} \mathbf{M}_{i}=\mathbf{0}
$$

By developing the balance of torques (the interested reader can refer to [3]), it finally results in:

$$
\boldsymbol{\omega}=(\mathbf{I}-\mathbf{c})^{-1} \cdot(\boldsymbol{\epsilon}:(\nabla \mathbf{v} \cdot \mathbf{c})),
$$

where $\omega$ represents the cluster angular velocity and $\mathbf{c}$ is the conformation tensor defined from

$$
\mathbf{c}=\frac{\sum_{i=1}^{i=N} S_{i}^{2} \mathbf{q}_{i} \otimes \mathbf{q}_{i}}{\sum_{i=1}^{i=N} S_{i}^{2}} .
$$

An extremely important consequence of this analysis is that rigid clusters composed of rods having the same conformation tensor $\mathbf{c}$ rotate at the same angular velocity.

As the conformation tensor $\mathbf{c}$ is symmetric and positive definite, it has real eigenvalues and eigenvectors. In $3 \mathrm{D}$, the three mutually perpendicular eigenvectors will be denoted by $\mathbf{u}_{1}, \mathbf{u}_{2}$, and $\mathbf{u}_{3}$, with the associated eigenvalues $\tau_{1}, \tau_{2}$, and $\tau_{3}$, respectively.

Imagine a rigid cluster composed of three rods oriented in directions $\mathbf{u}_{1}, \mathbf{u}_{2}$, and $\mathbf{u}_{3}$ with respective lengths $\sqrt{\tau_{1}}, \sqrt{\tau_{2}}$, and $\sqrt{\tau_{3}}$. The conformation tensor of such a three-rod cluster coincides with $\mathbf{c}$, and then both tensors have the same rotary velocity. Thus, in [3], the link between a rigid cluster composed of three mutually orthogonal rods and the Jeffery's triaxial ellipsoid has been proven. 


\subsection{Deformable clusters}

In the case of deformable clusters, two types of forces were assumed acting on each bead of a generic rod $\mathcal{R}$ (of length $2 L$ ) [8].

One, $\mathbf{F}_{i}^{H}$, is due to the fluid-rod friction once again modelled as

$$
\mathbf{F}_{i}^{H}=\xi\left(\mathbf{v}_{0}^{i}+\nabla \mathbf{v} \cdot \mathbf{p}_{i} L-\mathbf{v}_{G}^{i}-\dot{\mathbf{p}}_{i} L\right)
$$

where the superscript " $H$ " refers to its hydrodynamic nature, $\mathbf{v}_{0}^{i}$ is the fluid velocity (assumed unperturbed by the presence of the cluster) at the rod $\mathcal{R}_{i}$ centre of gravity $\mathcal{G}_{i}$ and $\mathbf{v}_{G}^{i}$ the velocity of the rod $\mathcal{R}_{i}$ centre of gravity $\mathcal{G}_{i}$. The other one, $\mathbf{F}_{i}^{C}$, is due to the rods entanglements. That force is assumed scaling with the difference between the rigid motion velocity (the one that the bead would have if the cluster would be rigid) $\mathbf{v}_{\mathcal{B}_{i}}^{R}$ and the real one $\mathbf{v}_{G}^{i}+\dot{\mathbf{p}}_{i} L$.

As proven in the previous section, the bead velocity when assuming the cluster rigid reads:

$$
\mathbf{v}_{\mathcal{B}_{i}}^{R}=\mathbf{V}_{G}+\mathbf{W} \cdot S_{i} \mathbf{q}_{i}=\mathbf{V}_{0}+\mathbf{W} \cdot \mathbf{r}_{i}+\mathbf{W} \cdot \mathbf{p}_{i} L,
$$

where the fact that $\mathbf{V}_{G}=\mathbf{V}_{0}$ was taken into account. In the previous equation, $\mathbf{r}_{i}$ is the vector connecting the cluster centre of gravity $\mathbf{G}$ to the rod centre of gravity $\mathcal{G}_{i}$, i.e., $\mathbf{r}_{i}=\mathcal{G}_{i}-\mathbf{G}$ and tensor $\mathbf{W}$ derives from the rigid rotary velocity $\omega$ given by Eq. (6) from

$$
\mathbf{W} \cdot \mathbf{p}_{i}=\omega \times \mathbf{p}_{i}, \quad \forall \mathbf{p}_{i} .
$$

Thus, the contribution to the bead force due to collective effects (a sort of mean field) when considering the generic $\operatorname{rod} \mathcal{R}_{i}$ reads:

$$
\mathbf{F}_{i}^{C}=\mu\left(\mathbf{V}_{0}+\mathbf{W} \cdot \mathbf{r}_{i}+\mathbf{W} \cdot \mathbf{p}_{i} L-\mathbf{v}_{G}^{i}-\dot{\mathbf{p}}_{i} L\right),
$$

and the net force acting on the bead located at $\mathcal{G}_{i}+\mathbf{p}_{i} L$ is given by $\mathbf{F}_{i}\left(\mathcal{G}_{i}+\mathbf{p}_{i} L\right)=\mathbf{F}_{i}^{H}\left(\mathcal{G}_{i}+\mathbf{p}_{i} L\right)+\mathbf{F}_{i}^{C}\left(\mathcal{G}_{i}+\right.$ $\left.\mathbf{p}_{i} L\right)$.

The linear momentum balance, neglecting inertia effects, allows us to obtain the velocity of the rods centre of gravity [8]

$$
\mathbf{v}_{G}^{i}=\mathbf{V}_{0}+\frac{\xi}{\xi+\mu} \nabla \mathbf{v} \cdot \mathbf{r}_{i}+\frac{\mu}{\xi+\mu} \mathbf{W} \cdot \mathbf{r}_{i}
$$

Now, the angular momentum balance leads to the rods rotary velocity (the interested reader can refer to [8] for the exhaustive derivation)

$$
\dot{\mathbf{p}}_{i}=\frac{\xi}{\xi+\mu} \dot{\mathbf{p}}_{i}^{J}+\frac{\mu}{\xi+\mu} \mathbf{W} \cdot \mathbf{p}_{i}=\frac{\xi}{\xi+\mu} \dot{\mathbf{p}}_{i}^{J}+\frac{\mu}{\xi+\mu} \dot{\mathbf{p}}_{i}^{R},
$$

where $\dot{\mathbf{p}}_{i}^{J}$ represents the orientation evolution of a rod in the absence of collective effects (dilute regime described by the Jeffery's equation) and $\dot{\mathbf{p}}_{i}^{R}$ the one of a rod in a rigid cluster.

It can be noticed that when $\xi \gg \mu$, hydrodynamic effects are preponderant and the rod kinematics are governed by the Jeffery's equation, i.e., $\dot{\mathbf{p}}_{i} \approx \dot{\mathbf{p}}_{i}^{J}$. In the opposite case, $\mu \gg \xi$, the cluster is too rigid and the rods adopt the velocity dictated by the rigid cluster kinematics $\dot{\mathbf{p}}_{i} \approx \dot{\mathbf{p}}_{i}^{R}$.

The main macroscopic descriptors of a deformable cluster are its shape and its microstructure.

\subsubsection{Macroscopic description: clusters inertia and shape}

Integrating the position of the centre of gravity of each rod composing the cluster from Eq. (12), it is natural to obtain the cluster inertia and shape.

The cluster inertia tensor $\mathbf{S}$ is defined from a continuous description of the cluster: the rods individuality is replaced by a distribution function $\psi^{r}(\mathbf{r})$ and then integrated:

$$
\mathbf{S}=\int_{\mathcal{S}} \mathbf{r} \otimes \mathbf{r} \psi^{r}(\mathbf{r}) \mathrm{d} \mathbf{r}
$$


Its time evolution, according to [8], results in

$$
\dot{\mathbf{S}}=\frac{\xi}{\xi+\mu}\left(\nabla \mathbf{v} \cdot \mathbf{S}+\mathbf{S} \cdot(\nabla \mathbf{v})^{\mathrm{T}}\right)+\frac{\mu}{\xi+\mu}(\mathbf{W} \cdot \mathbf{S}-\mathbf{S} \cdot \mathbf{W}) .
$$

The trace of $\mathbf{S}$ gives an information on the cluster size. A normalized inertia tensor-called shape tensor s-can be defined as:

$$
\mathbf{s}=\frac{\mathbf{S}}{\operatorname{Tr}(\mathbf{S})} .
$$

\subsubsection{Macroscopic description: orientation}

Now, if the orientation of the rods of a deformable cluster is assumed to be given by the orientation distribution $\psi^{p}(\mathbf{p})$, the time derivative of its second-order moment a yields [8]:

$$
\dot{\mathbf{a}}=\frac{\xi}{\xi+\mu} \dot{\mathbf{a}}^{J}+\frac{\mu}{\xi+\mu} \dot{\mathbf{a}}^{R},
$$

whose objectivity was proven in [15], and where $\dot{\mathbf{a}}^{J}$ and $\dot{\mathbf{a}}^{R}$ are given by

$$
\left\{\begin{array}{l}
\dot{\mathbf{a}}^{J}=\nabla \mathbf{v} \cdot \mathbf{a}+\mathbf{a} \cdot(\nabla \mathbf{v})^{\mathrm{T}}-2 \mathbf{A}: \mathbf{D} \\
\dot{\mathbf{a}}^{R}=\mathbf{W} \cdot \mathbf{a}+\mathbf{a} \cdot \mathbf{W}^{\mathrm{T}}
\end{array}\right.
$$

with A the fourth-order orientation tensor expressed from the second-order one by considering any of the numerous closure relations proposed in the literature $[4,21,31,40]$.

It has just been proven that the conformation of a deformable cluster is defined from both the orientation tensor $\mathbf{a}$ and the inertia tensor $\mathbf{S}$. The evolution of both tensors makes use of tensor $\mathbf{W}$ that depends itself on the conformation tensor $\mathbf{c}$. Tensor $\mathbf{c}$ is very close to tensor $\mathbf{S}$; the first is defined from the vectors joining the cluster centre of gravity with each rod bead, whereas the second one involves vectors joining the cluster centre of gravity with the rods centre of gravity. If the length of the rods is very small compared to the cluster size, both tensors are very close and consequently tensor $\mathbf{W}$ can be evaluated by using $\mathbf{s}$ instead of $\mathbf{c}$. In [8], the following expression was obtained:

$$
\mathbf{c}=\frac{\mathbf{S}+L^{2} \mathbf{a}}{\operatorname{Tr}(\mathbf{S})+L^{2}},
$$

that as soon as $L^{2} \ll \operatorname{Tr}(\mathbf{S})$ leads to $\mathbf{c} \approx \mathbf{s}$.

\section{Fine-scale discrete simulation}

In order to compute the reference solution of a moving cluster suspending in a fluid flow, we proceed with a direct numerical simulation based on a molecular dynamics representation of the entangled rods composing a dense cluster. This modelling approach is based on the following assumptions: (1) each of the R rods consists of a set of $\mathrm{P}^{\Upsilon}$ connected particles (the total number of particles in the system being $\mathrm{P}$ ); (2) particles interactions are described from appropriate potentials, in particular, the Lennard-Jones potential $V^{L J}$ used to describe the inter-particles interactions (and consequently ensuring inter-rods interaction), and two other potentials, $V^{E}$ and $V^{B}$, used to describe the rod elongation and bending, respectively, see Fig. 2. Thus, the total potential reads

$$
V=\sum_{j=1}^{\mathrm{R}} \sum_{i=1}^{\mathrm{P}^{\mathrm{r}}-1} V_{i, i+1 ; j}^{E}+\sum_{j=1}^{\mathrm{R}} \sum_{i=2}^{\mathrm{P}^{\mathrm{r}}-1} V_{i-1, i, i+1 ; j}^{B}+\sum_{i=1}^{\mathrm{P}} \sum_{k>i}^{\mathrm{P}} V_{k \notin \mathcal{R}(i)}^{L J}
$$

where $j$ refers to the rods $\mathcal{R}_{j}, \mathcal{R}(i)$ to the rod to which particle $i$ belongs, and indexes $i$ and $k$ are related to particles $\mathcal{P}_{i}$ and $\mathcal{P}_{k}$. Index $j$ in potentials $V_{i, i+1 ; j}^{E}$ and $V_{i-1, i, i+1 ; j}^{B}$ indicates that particle $\mathcal{P}_{i}$ belongs to rod $\mathcal{R}_{j}$.

The Lennard-Jones potential reads

$$
V_{i, k}^{L J}=4 \varepsilon\left(\left(\frac{\sigma}{d_{i k}}\right)^{12}-\left(\frac{\sigma}{d_{i k}}\right)^{6}\right),
$$




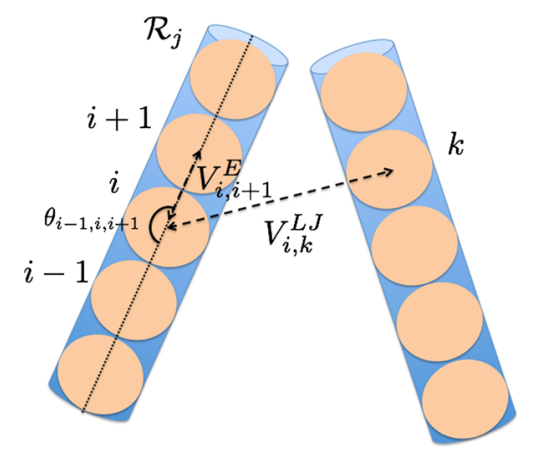

Fig. 2 Sketch of rods, particles and potentials

with $d_{i k}=\left\|\mathbf{x}_{k}-\mathbf{x}_{i}\right\|$ the distance between particles $\mathcal{P}_{i}$ and $\mathcal{P}_{k}$, located at positions $\mathbf{x}_{i}$ and $\mathbf{x}_{k}$, respectively, and $\epsilon$ and $\sigma$ the two usual parameters involved in the Lennard-Jones potential.

The elongation potential is given by

$$
V_{i, i+1}^{E}=\frac{K_{E}}{2}\left(1-\frac{d_{i j}}{d_{\mathrm{eq}}}\right)^{2}
$$

where $d_{\mathrm{eq}}$ is the equilibrium distance between two particles, distance at which the potential reaches its minimum (and consequently the associated force vanishes). In the previous expression, $K_{E}$ reflects the potential intensity.

The bending potential between three successive particles belonging to the same fibre reads

$$
V_{i-1, i, i+1}^{B}=\frac{K_{B}}{2}\left(\theta_{i-1, i, i+1}-\theta_{\mathrm{eq}}\right)^{2},
$$

where $\theta_{i-1, i, i+1}$ is the angle defined by vectors joining particles $i-1, i$ and $i, i+1$ with $\theta_{\text {eq }}$ the angle at equilibrium. In our study, we consider without loss of generality $\theta_{\mathrm{eq}}=0$. Again, the potential intensity is described from $K_{B}$.

Force applying at each particle results from the potential derivative according to

$$
\mathbf{F}_{i}^{I}=-\frac{\partial V}{\partial \mathbf{x}_{i}} .
$$

The other force acting on each particle is the one related to the fluid $\operatorname{drag} \mathbf{F}_{i}^{D}$. Let $\mathbf{v}(\mathbf{x})$ be the fluid velocity at position $\mathbf{x}$, assumed unperturbed by the rods presence, the drag force reads

$$
\mathbf{F}_{i}^{D}=\xi\left(\mathbf{v}\left(\mathbf{x}_{i}\right)-\dot{\mathbf{x}}_{i}\right)
$$

where $\dot{\mathbf{x}}_{i}$ denotes the velocity of particle $\mathcal{P}_{i}$.

The linear momentum balance involving particle $\mathcal{P}_{i}$ reads

$$
\mathbf{F}_{i}=\mathbf{F}_{i}^{D}+\mathbf{F}_{i}^{I}=m \mathbf{a}_{i},
$$

with the particle acceleration $\mathbf{a}_{i}=\frac{\mathrm{d} \dot{\mathbf{x}}_{i}}{\mathrm{~d} t}=\frac{\mathrm{d}^{2} \mathbf{x}_{i}}{\mathrm{~d} t^{2}}$.

A second-order time integration scheme is considered, consisting of

$$
\left\{\begin{array}{l}
\mathbf{a}_{i}^{n+1}=\frac{\mathbf{F}_{i}^{n}}{m} \\
\mathbf{x}_{i}^{n+1}=\mathbf{x}_{i}^{n}+\dot{\mathbf{x}}_{i}^{n} \Delta t+\frac{1}{2} \mathbf{a}_{i}(t)(\Delta t)^{2}, \\
\dot{\mathbf{x}}_{i}^{n+1}=\dot{\mathbf{x}}_{i}^{n}+\frac{1}{2}\left(\mathbf{a}_{i}^{n}+\mathbf{a}_{i}^{n+1}\right) \Delta t
\end{array},\right.
$$

where the superscript $n$ refers to the time step, $t_{n}=n \Delta t$.

Prior to proceed with the time integration, equations are rewritten in dimensionless form, by considering as characteristic variables 

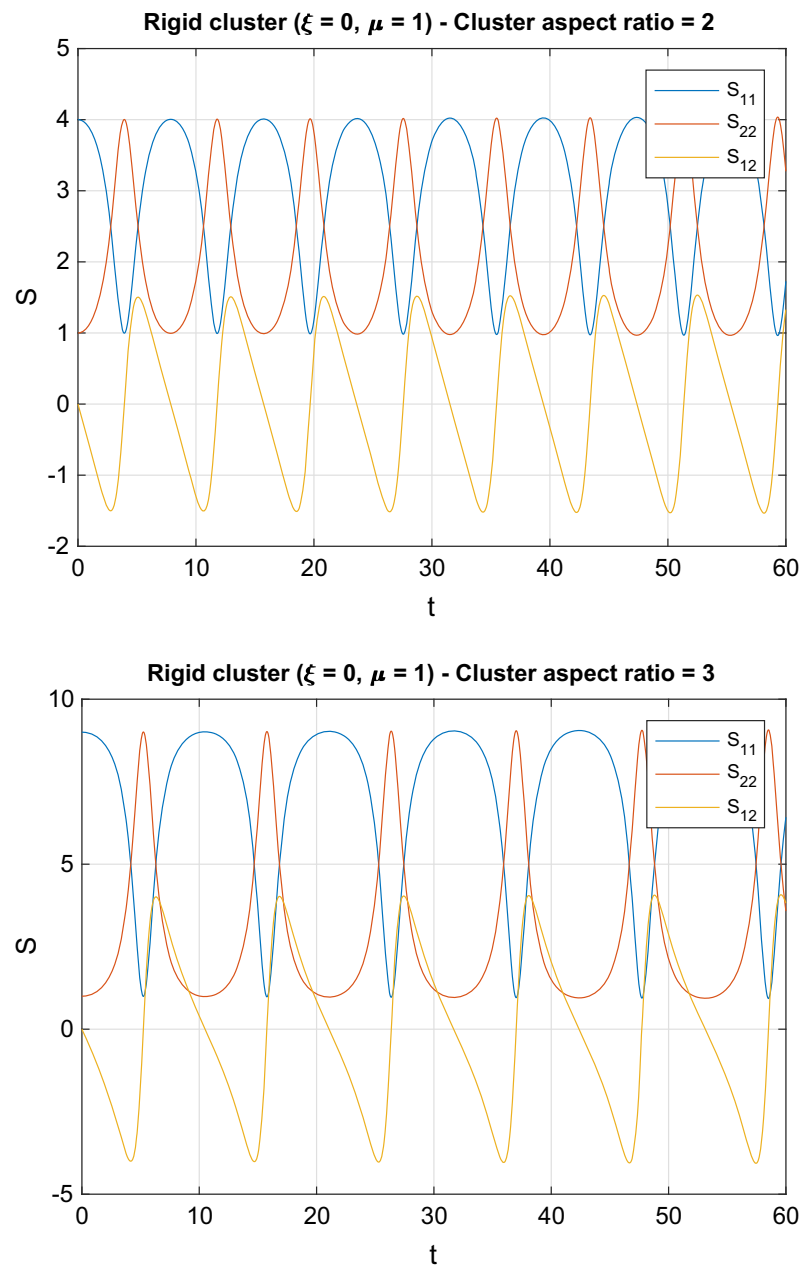

Fig. 3 Rigid clusters with different aspect ratio

- the length: $\sigma$

- the energy: $\varepsilon$

- the mass: $m$

- the velocity: $\sqrt{\varepsilon / m}$

- the acceleration: $\varepsilon /(m \sigma)$

- the time: $\sigma \sqrt{m / \varepsilon}$

that allows writing the dimensionless model in which for the sake of clarity, we do not change the notation for designating the dimensionless variables.

Thus, we obtain

$$
\left\{\begin{array}{l}
V_{i, j}=4\left(\left(\frac{1}{d_{i j}}\right)^{12}-\left(\frac{1}{d_{i j}}\right)^{6}\right) \\
V_{i, i+1}^{E}=\frac{\hat{K}_{E}}{2}\left(1-\frac{d_{i j}}{d_{\mathrm{eq}}}\right)^{2} \\
V_{i-1, i, i+1}^{B}=\frac{\hat{K}_{B}}{2}\left(\theta_{i-1, i, i+1}-\theta_{\mathrm{eq}}\right)^{2} \\
\mathbf{F}_{i}^{I}=-\frac{\partial V}{\partial \mathbf{x}_{i}} \\
\mathbf{F}_{i}^{D}=\hat{\xi}\left(\mathbf{v}\left(\mathbf{x}_{i}\right)-\dot{\mathbf{x}}_{i}\right) \\
\mathbf{F}_{i}=\mathbf{a}_{i}
\end{array}\right.
$$



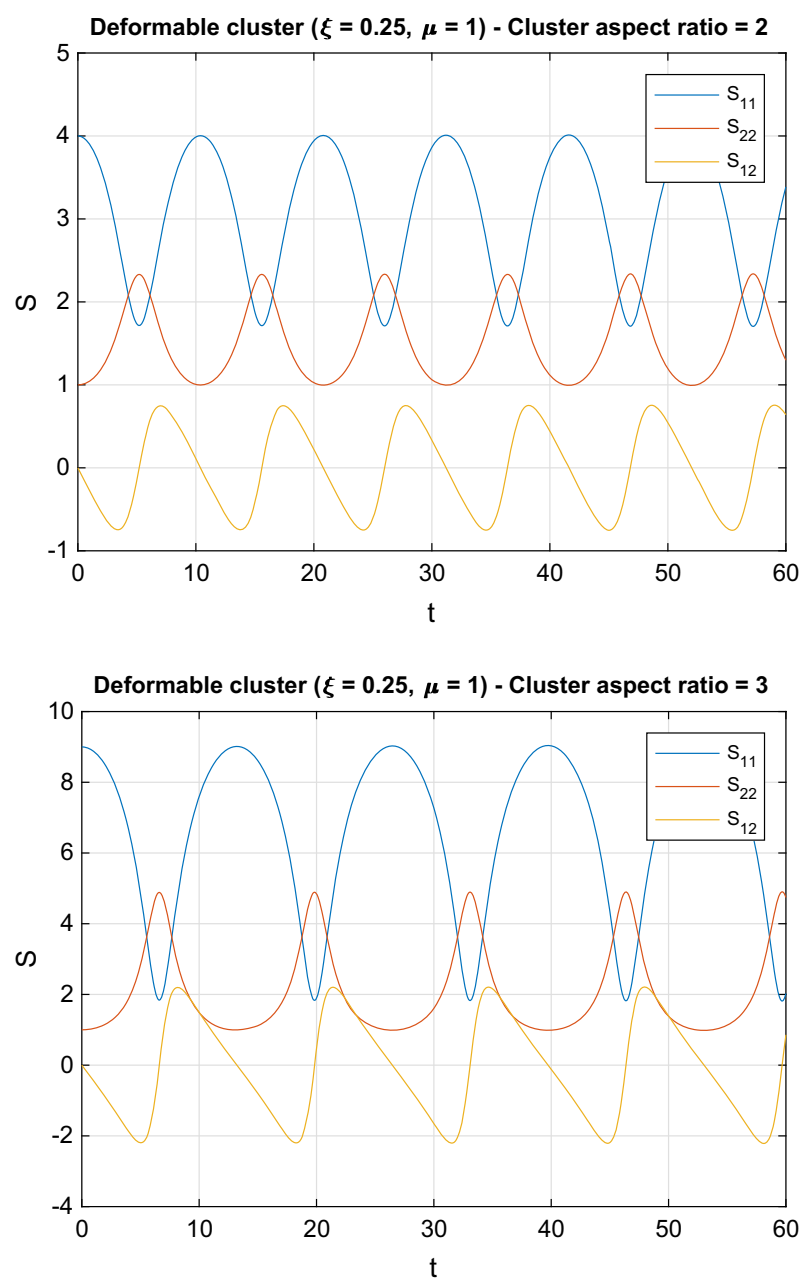

Fig. 4 Deformable clusters with different aspect ratio

where $\hat{K}_{E}=\frac{K_{E}}{\varepsilon}, \hat{K}_{B}=\frac{K_{B}}{\varepsilon}, \hat{\xi}=\xi \sqrt{\varepsilon m} / \sigma$, with $\hat{K}_{B}=100, \hat{K}_{E}=100$, and the dimensionless equilibrium distance $d_{\text {eq }}=2^{1 / 6}$. The applied flow consists of a simple shear flow with dimensionless shear rate $\dot{\gamma}=5$.

Thus, at the microscopic scale, as soon as $K_{E}$ and $K_{B}$ are taken large enough to represent almost nondeformable rods, the model only contains one parameter that controls numerical simulations, $\hat{\xi}$.

\section{Numerical results}

In this section, we first present numerical simulations to illustrate the behaviour of the proposed model and then a comparison of the model with fine-scale direct simulations.

The equations governing the rate of change of the cluster orientation and inertia tensors, Eqs. (17) and (15) respectively, have similar expressions. They contain a term, the last one, that introduces the mean field effects, in particular the cluster rotation, scaling with tensor $\mathbf{W}$ that in turn depends on the cluster shape.

In order to better understand the underlying physics of those equations, we are considering both rigid and deformable clusters of different aspect ratio and evaluating the time evolution of their inertia tensor.

Firstly, Fig. 3 compares for two rigid clusters of different aspect ratio the time evolution of their inertia tensor $\mathbf{S}$. As expected from our previous works [3], inertia tensor is simply rotated with a period that scales with the cluster aspect ratio, i.e. the higher the cluster aspect ratio, the larger the rotation period. 

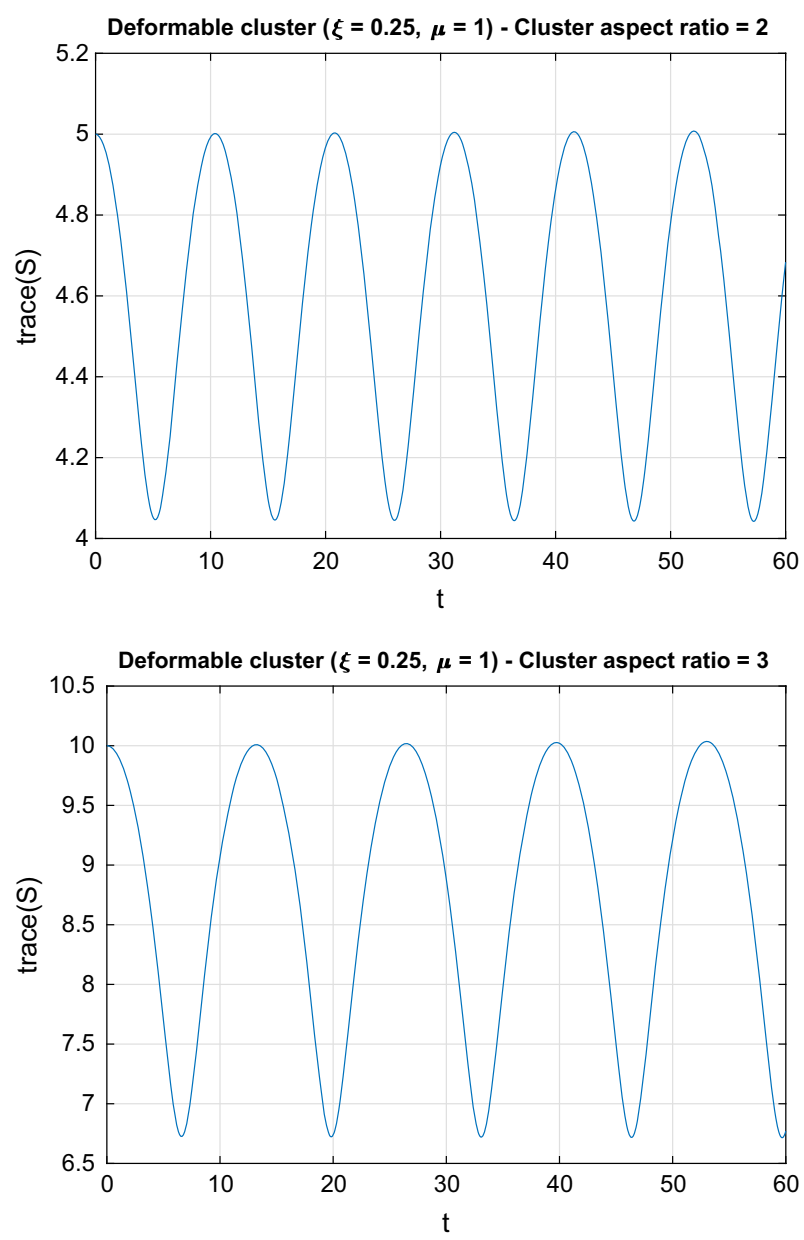

Fig. 5 Inertia tensor trace time evolution

When examining the evolution of the trace of the inertial tensor over time, it can be noticed that it remains constant.

Secondly, Fig. 4 presents similar results but now for deformable cluster with the same initial aspect ratios. It can be noticed that inertial tensor remains periodic with a similar evolution of its period with respect to the cluster aspect ratio. Moreover, the cluster deformability results in a periodic evolution of the tensor trace, as Fig. 5 reveals. Thus, the cluster is stretched and then compressed during a complete rotation, while keeping its perfect periodicity. Moreover, as expected, the higher the cluster aspect ratio, the larger the amplitude of the inertia tensor trace evolution.

Figure 6 compares now the model predictions with direct simulation. The applied flow and the considered inter-rods interaction potential almost guarantee the cluster integrity, that is, the cluster rotates while slightly deforming, but after a complete rotation, the initial configuration is almost recovered. In this figure, it can be noticed that direct numerical simulation exhibits some amount of irreversible cluster deformation, with the consequent impact on the cluster rotary velocity and in its period of rotation, increasing as the cluster aspect ratio enlarges. Both solutions are in good agreement.

Figure 7 compares, for the aggregate considered in Fig. 6, the prediction of the model for a deformable cluster with the prediction obtained by assuming the cluster is rigid. It can be noticed that as discussed above, cluster deformability induces a slight increase in the rotation period.

Figure 8 compares model prediction and direct simulation for larger shear rates (larger with respect to the rods interaction preserving the aggregate integrity). The model prediction differs from direct simulation since the applied shear rates produce cluster degradation, as evidenced in Fig. 9, where some rods detach from the cluster. This scenario compromises the validity of the proposed macroscopic model and defines the limit of its interval of validity. 

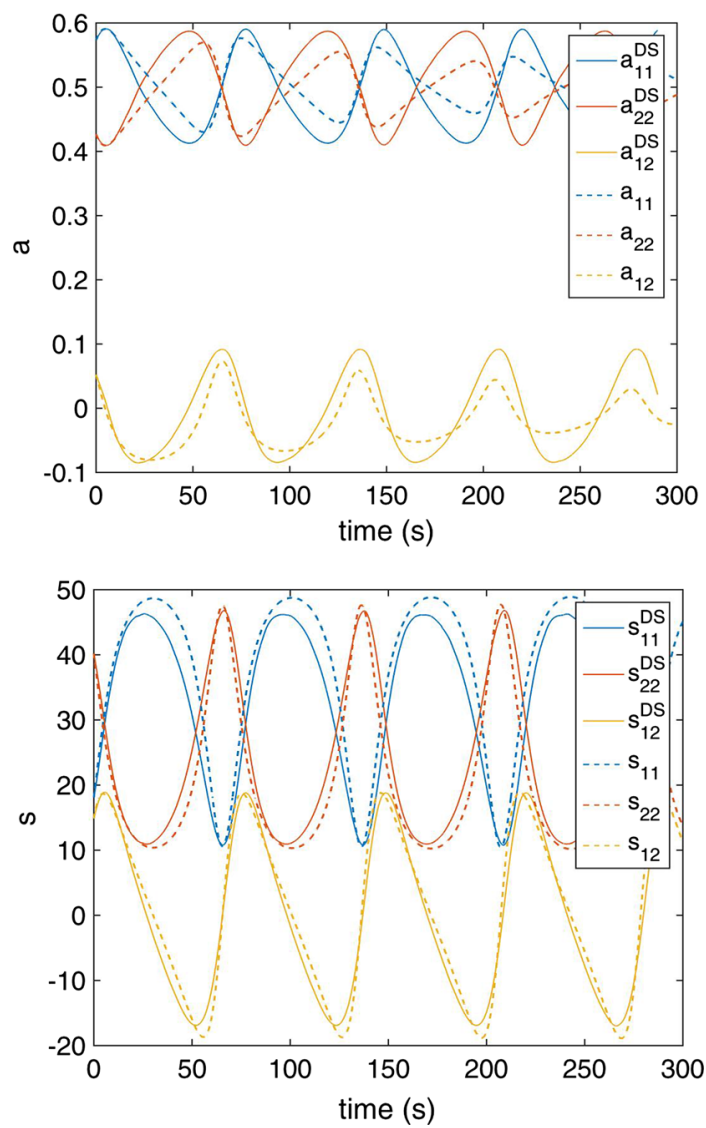

Fig. 6 Comparison of cluster orientation and inertia evolution: model prediction (dashed line) versus direct simulations

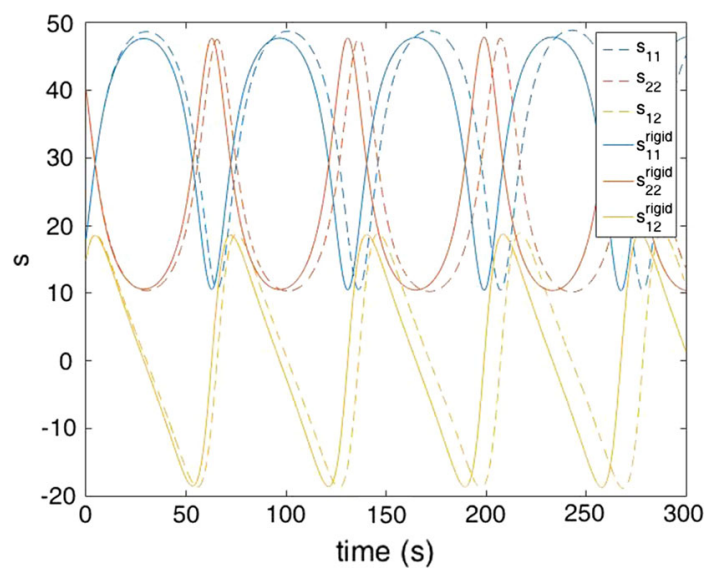

Fig. 7 Comparison of cluster orientation and inertia evolution: deformable versus rigid cluster model

\section{Conclusions}

In this work, a multi-scale model of deformable clusters, based on the use of two tensors, the first associated with the global cluster shape and the second with the orientation of the rods composing, is presented and its predictions are compared with finer descriptions inspired from molecular dynamics.

Using fine-scale simulations, it was proven that clusters deform while rotating, and it was found that the model almost captures the shape and orientation evolution. When the applied shear rate becomes too large, rods 

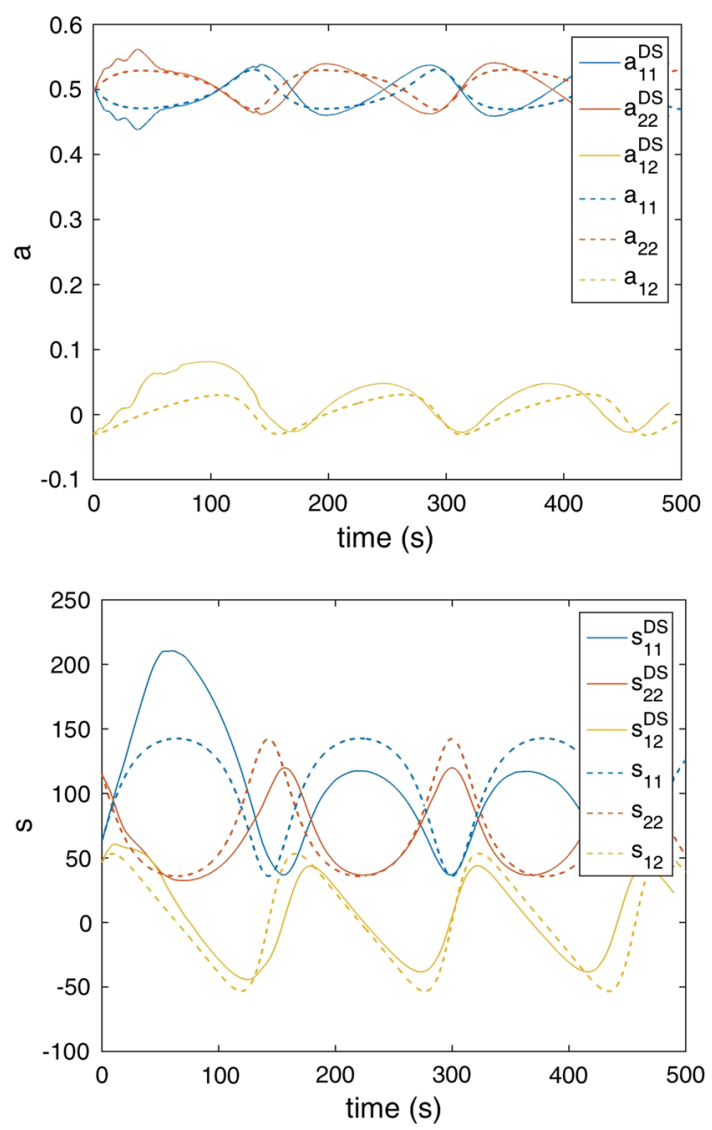

Fig. 8 Comparison of cluster orientation and inertia evolution: model prediction (dashed line) versus direct simulations for larger applied shear rates

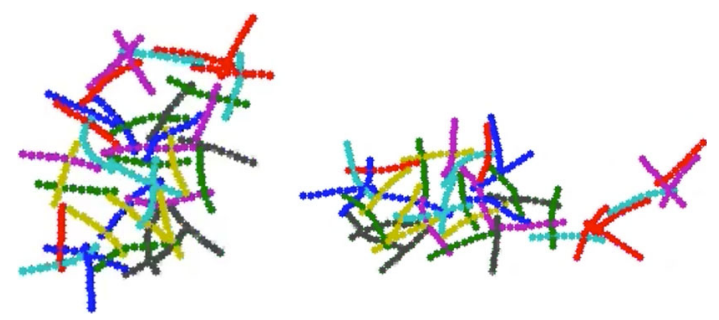

Fig. 9 Aggregate conformation: (left) initial configuration and (right) configuration after a first rotation

detach from the clusters and model predictions exhibit significant differences with respect to direct numerical simulations.

Compliance with ethical standards

Conflict of interest The authors declare that they have no conflict of interest.

\section{References}

1. Abisset-Chavanne, E., Mezher, R., Le Corre, S., Ammar, A., Chinesta, F.: Kinetic theory microstructure modeling in concentrated suspensions. Entropy 15, 2805-2832 (2013)

2. Abisset-Chavanne, E., Ferec, J., Ausias, G., Cueto, E., Chinesta, F., Keunings, R.: A second-gradient theory of dilute suspensions of flexible rods in a Newtonian fluid. Arch. Comput. Methods Eng. 22, 511-527 (2015) 
3. Abisset-Chavanne, E., Chinesta, F., Ferec, J., Ausias, G., Keunings, R.: On the multiscale description of dilute suspensions of non-Brownian rigid clusters composed of rods. J. Non Newton. Fluid Mech. 222, 34-44 (2015)

4. Advani, S., Tucker, C.: Closure approximations for three-dimensional structure tensors. J. Rheol. 34, 367-386 (1990)

5. Ammar, A., Mokdad, B., Chinesta, F., Keunings, R.: A new family of solvers for some classes of multidimensional partial differential equations encountered in kinetic theory modeling of complex fluids. J. Non Newt. Fluid Mech. 139, 153-176 (2006)

6. Ammar, A., Mokdad, B., Chinesta, F., Keunings, R.: A new family of solvers for some classes of multidimensional partial differential equations encountered in kinetic theory modeling of complex fluids. Part II: transient simulation using space-time separated representations. J. Non Newt. Fluid Mech. 144, 98-121 (2007)

7. Batchelor, G.K.: The stress system in a suspension of force-free particles. J. Fluid Mech. 41, 545-570 (1970)

8. Binetruy, C., Chinesta, F., Keunings, R.: Flows in Polymers, Reinforced Polymers and Composites. A Multi-scale Approach. Springer Briefs in Applied Sciences and Technology. Springer, Berlin (2015)

9. Bird, R.B., Curtiss, C.F., Armstrong, R.C., Hassager, O.: Dynamic of Polymeric Liquid, Volume 2: Kinetic Theory. Wiley, New York (1987)

10. Chaubal, C.V., Srinivasan, A., Egecioglu, O., Leal, L.G.: Smoothed particle hydrodynamics techniques for the solution of kinetic theory problems. J. Non Newton. Fluid Mech. 70, 125-154 (1997)

11. Chauviere, C., Lozinski, A.: Simulation of dilute polymer solutions using a Fokker-Planck equation. Comput. Fluids 33, 687-696 (2004)

12. Chiba, K., Ammar, A., Chinesta, F.: On the fiber orientation in steady recirculating flows involving short fibers suspensions. Rheol. Acta 44, 406-417 (2005)

13. Chinesta, F., Chaidron, G., Poitou, A.: On the solution of the Fokker-Planck equation in steady recirculating flows involving short fibre suspensions. J. Non Newton. Fluid Mech. 113, 97-125 (2003)

14. Chinesta, F., Ammar, A., Leygue, A., Keunings, R.: An overview of the Proper Generalized Decomposition with applications in computational rheology. J. Non Newton. Fluid Mech. 166, 578-592 (2011)

15. Chinesta, F.: From single-scale to two-scales kinetic theory descriptions of rods suspensions. Arch. Comput. Methods Eng. 20(1), 1-29 (2013)

16. Chinesta, F., Keunings, R., Leygue, A.: The Proper Generalized Decomposition for Advanced Numerical Simulations. A Primer Springerbriefs. Springer, Berlin (2014)

17. Cruz, C., Illoul, L., Chinesta, F., Regnier, G.: Effects of a bent structure on the linear viscoelastic response of Carbon Nanotube diluted suspensions. Rheol. Acta 49, 1141-1155 (2010)

18. Cruz, C., Chinesta, F., Regnier, G.: Review on the Brownian dynamics simulation of bead-rod-spring models encountered in computational rheology. Arch. Comput. Methods Eng. 19(2), 227-259 (2012)

19. dell'Isola, F., Giorgio, I., Pawlikowski, M., Rizzi, N.L.: Large deformations of planar extensible beams and pantographic lattices: heuristic homogenization, experimental and numerical examples of equilibrium. Proc. R. Soc. Lond. A 472(2185), 20150790 (2016)

20. Doi, M., Edwards, S.F.: The Theory of Polymer Dynamics. Clarendon Press, Oxford (1987)

21. Dupret, F., Verleye, V.: Modelling the flow of fibre suspensions in narrow gaps. In: Siginer, D.A., De Kee, D., Chabra, R.P. (eds.) Advances in the Flow and Rheology of Non-Newtonian Fluids. Rheology Series, pp. 1347-1398. Elsevier, Amsterdam (1999)

22. Eremeyev, V.A., Lebedev, L.P., Altenbach, H.: Foundations of Micropolar Mechanics. Springer, Berlin (2012)

23. Folgar, F., Tucker, C.: Orientation behavior of fibers in concentrated suspensions. J. Reinf. Plast. Comp. 3, 98-119 (1984)

24. Hand, G.L.: A theory of anisotropic fluids. J. Fluid Mech. 13, 33-62 (1962)

25. Hinch, J., Leal, G.: The effect of Brownian motion on the rheological properties of a suspension of non-spherical particles. J. Fluid Mech. 52, 683-712 (1972)

26. Hinch, J., Leal, G.: Constitutive equations in suspension mechanics. Part I. J. Fluid Mech. 71, 481-495 (1975)

27. Hinch, J., Leal, G.: Constitutive equations in suspension mechanics. Part II. J. Fluid Mech. 76, 187-208 (1976)

28. Jeffery, G.B.: The motion of ellipsoidal particles immersed in a viscous fluid. Proc. R. Soc. Lond. A102, 161-179 (1922)

29. Keunings, R.: On the Peterlin approximation for finitely extensible dumbells. J. Non-Newton. Fluid Mech. 68, 85-100 (1997)

30. Keunings, R.: Micro-macro methods for the multiscale simulation viscoelastic flow using molecular models of kinetic theory. In: Binding, D.M., Walters, K. (eds.) Rheology Reviews, pp. 67-83, British Society of Rheology, Aberystwyth, UK (2004). http://www.bsr.org.uk/rheology_review.asp

31. Kroger, M., Ammar, A., Chinesta, F.: Consistent closure schemes for statistical models of anisotropic fluids. J. Nonnewton. Fluid Mech. 149, 40-55 (2008)

32. Lozinski, A., Chauviere, C.: A fast solver for Fokker-Planck equation applied to viscoelastic flows calculations: 2D FENE model. J. Comput. Phys. 189, 607-625 (2003)

33. Ma, A., Chinesta, F., Mackley, M., Ammar, A.: The rheological modelling of carbon nanotube (CNT) suspensions in steady shear flows. Int. J. Mater. Form. 2, 83-88 (2008)

34. Ma, A., Chinesta, F., Ammar, A., Mackley, M.: Rheological modelling of carbon nanotube aggregate suspensions. J. Rheol. 52(6), 1311-1330 (2008)

35. Ma, A., Chinesta, F., Mackley, M.: The rheology and modelling of chemically treated carbon nanotube suspensions. J. Rheol. 53(3), 547-573 (2009)

36. Mokdad, B., Pruliere, E., Ammar, A., Chinesta, F.: On the simulation of kinetic theory models of complex fluids using the Fokker-Planck approach. Appl. Rheol. 17/2, 26494, 1-14 (2007)

37. Öttinger, H.C., Laso, M.: Smart polymers in finite element calculation. In: International Congress on Rheology, Brussel (1992)

38. Petrie, C.: The rheology of fibre suspensions. J. Non-Newton. Fluid Mech. 87, 369-402 (1999)

39. Pietraszkiewicz, W., Eremeyev, V.A.: On natural strain measures of the non-linear micropolar continuum. Int. J. Solids Struct. 46(3-4), 774-787 (2009) 
40. Pruliere, E., Ammar, A., El Kissi, N., Chinesta, F.: Recirculating flows involving short fiber suspensions: numerical difficulties and efficient advanced micro-macro solvers. Arch. Comput. Methods Eng. State Art Rev. 16, 1-30 (2009)

41. Wapperom, P., Keunings, R.: Numerical simulation of branched polymer melts in transient complex flows using pom-pom models. J. Non Newton. Fluid Mech. 97, 267-281 (2001) 